\title{
Sets of Co-regulated Serum Lipids are Associated with Alzheimer Disease Pathophysiology
}

Dinesh Kumar Barupal ${ }^{1}$, Rebecca Bailli², Sili Fan'1 , Andrew J. Saykin ${ }^{3,4,5}$, Peter J.Meikle ${ }^{6}$, Matthias Arnold ${ }^{7,8}$, Kwangsik $\mathrm{Nho}^{3,4}$, Oliver Fiehn ${ }^{1^{\star}}$ and Rima KaddurahDaouk $^{9^{*}}$ and for the Alzheimer's Disease Neuroimaging Initiative ${ }^{10}$ and the Alzheimer Disease Metabolomics Consortium ${ }^{11}$

${ }^{1} \mathrm{NIH}-$ West Coast Metabolomics Center, University of California, Davis, 451 Health Sciences Drive, Davis, CA 95616, United States

${ }^{2}$ Rosa \& Co LLC, San Carlos, CA, USA

${ }^{3}$ Center for Neuroimaging, Department of Radiology and Imaging Sciences, Indiana University School of Medicine, Indianapolis, IN ${ }^{4}$ Indiana Alzheimer Disease Center, Indiana University School of Medicine, Indianapolis, IN

${ }^{5}$ Medical and Molecular Genetics Department, Indiana University School of Medicine, Indianapolis, IN

${ }^{6}$ Metabolomics Laboratory, Baker Heart and Diabetes Institute, 75 Commercial Road, Melbourne, VIC 3004, Australia

${ }^{7}$ Institute of Bioinformatics and Systems Biology, Helmholtz Zentrum München, German Research Center for Environmental Health, Neuherberg, Germany

${ }^{8}$ German Center for Diabetes Research (DZD), Neuherberg, Germany

${ }^{9}$ Department of Psychiatry and Behavioral Sciences; Department of Medicine and the Duke Institute for Brain Sciences, Duke University, Durham, NC, USA

${ }^{10}$ Data used in preparation of this article were obtained from the Alzheimer's Disease Neuroimaging Initiative (ADNI) database (adni.loni.usc.edu). As such, the investigators within the ADNI contributed to the design and implementation of ADNI and/or provided data but did not participate in analysis or writing of this report. A complete listing of ADNI investigators can be found at: http://adni.loni.usc.edu/wpcontent/uploads/how to apply/ADNI Acknowledgement List.pdf

${ }^{11}$ Data used in preparation of this article were generated by the Alzheimer's Disease Metabolomics Consortium (ADMC). All authors are members of the ADMC. A complete listing of ADMC investigators can be found at: https://sites.duke.edu/adnimetab/aboutus/the-team/

\section{Address correspondence to:}

1. Oliver Fiehn

Email : ofiehn@ucdavis.edu Tel: +1-530-754-8258;

451 East Health Science Drive, Genome Center

University of California, Davis. 95616 CA USA

2. Rima Kaddurah Daouk

Duke University Medical Center, Durham, NC, USA

Phone +1-919-684-2611

Email: kaddu001@mc.duke.edu 
INTRODUCTION: Altered regulation of lipid metabolism in Alzheimer disease (AD) can be characterized using lipidomic profiling.

METHOD: 349 serum lipids were measured in 806 participants enrolled in the Alzheimer Disease Neuroimaging Initiative Phase 1 (ADNI1) cohort and analysed using lipid regression models and lipid set enrichment statistics.

RESULTS: AD diagnosis was associated with 7 of 28 lipid sets of which four also correlated with cognitive decline, including polyunsaturated fatty acids. CSF amyloid beta $A \beta_{1-42}$ correlated with glucosylceramides, lysophosphatidyl cholines and unsaturated triacylglycerides; CSF total tau and brain atrophy correlated with monounsaturated sphingomyelins and ceramides, in addition to EPA-containing lipids.

DISCUSSION: Lipid desaturation, elongation and acyl chain remodeling are dysregulated across the spectrum of AD pathogenesis. Monounsaturated lipids were important in early stages of $A D$, while polyunsaturated lipid metabolism was associated with later stages of $A D$.

\section{SIGNFICANCE}

Both metabolic genes and co-morbidity with metabolic diseases indicate that lipid metabolism is critical in the etiology of Alzheimer's disease (AD). For 800 subjects, we found that sets of blood lipids were associated with current AD-biomarkers and with AD clinical symptoms. Our study highlights the role of disturbed acyl chain lipid remodelling in several lipid classes. Our work has significant implications on finding a cure for AD. Depending on subject age, human blood lipids may have different effects on AD development. Remodelling of acyl chains needs to be studied in relation to genetic 
72 variants and environmental factors. Specifically, the impact of dietary supplements and

73 drugs on lipid remodelling must be investigated.

\section{ABBREVIATIONS}

$75 \quad$ FA - fatty acids

76 AC - acetyl carnitine

$77 \quad$ PC phosphatidylcholine

78 CE - cholesteryl esters

79 SM - sphingomyelins

80 Cer - Ceramide

81 PE - phosphatidylethanolamine

$82 \mathrm{TG}$ - triacylglycerol

$83 \mathrm{PI}$ - phosphatidylinositol

$84 \quad$ DG - diacylglycerol

85 LPC - lysophosphatidylcholine

$86 \quad A \beta-$ Amyloid Beta

87 SPARE-AD - Spatial Pattern of Abnormality for Recognition of Early Alzheimer's

88 disease

89 LCRS - Lipid Co-Regulatory Set

90 MUFA - mono unsaturated fatty acid

$91 \quad$ PUFA - poly unsaturated fatty acid

92 ADASCog13 - Alzheimer's Disease Assessment Scale-Cognitive subscale

$93 \quad \mathrm{ADNI}-\mathrm{Alzheimer's} \mathrm{disease} \mathrm{neuroimaging} \mathrm{initiative}$

94 EPA - eicosapentaenoic acid 
CSF - cerebrospinal fluid

\section{Introduction}

98

The failure of clinical trials of disease modifying agents in Alzheimer's disease (AD) highlights our limited knowledge about underlying pathophysiological mechanisms. AD often presents with diabetes co-morbidity and a wide range of metabolic perturbations occurring early in the disease process (1). APOE- $\varepsilon 4$ is by far the strongest single gene variant contributing to increased $A D$ risk and plays key roles in lipid transport and metabolism. Presence of the APOE- $\varepsilon 4$ variant is correlated with higher cholesterol levels in the blood (2). More than twenty additional genes have recently been implicated in AD with functional roles in lipid processing, immune regulation and phagocytosis. Hence, both co-morbidities and known gene variants support the idea that metabolic dysfunctions may contribute to AD onset and progression.

Lipidomics methods using liquid chromatography and mass spectrometry (LC/MS) yield reliable measurements of hundreds of lipids in biological samples. LC/MS methods have been used in AD studies in attempts to define possible risk factors (3-7), diagnostic markers (8) and for highlighting novel drug targets (9-11). Perturbations in ceramides and related sphingomyelin metabolism $(4,7)$ were noted in many of these studies pointing towards a possible role of these lipids in aberrant signalling pathways, membrane remodelling, and apoptotic cascades during AD progression.

Changes in phosphatidylcholines were observed in several studies (11-13) pointing to a possible role for phospholipid metabolism in $A D$ pathogenesis. Yet, AD risk prediction failed to replicate using a phosphatidylcholine (PC) biomarker panel(11, 14). Partial correlation network analysis indicated early AD biomarker $A \beta_{1-42}$ was associated with PC and sphingomyelin (SM) (11). These studies support our hypothesis that distinct lipid 
biochemical pathways were dysregulated in early and late phase of AD. Here, we used

LC-MS/MS based serum lipidomics analysis measured in the ADNI I cohort to define the has been successfully used in the analysis of transcriptomic data (15). We investigated correlation of lipid sets with (1) Disease diagnosis, (2) CSF markers of disease $A \beta_{1-42}$, CSF total tau and (3) cognitive decline and brain atrophy.

\section{Material and methods}

\subsection{Study cohort}

Data used in the preparation of this article were obtained from the Alzheimer's Disease

Neuroimaging Initiative (ADNI) database (adni.loni.usc.edu). The ADNI was launched in 2003 as a public-private partnership, led by Principal Investigator Michael W. Weiner, MD. The primary goal of ADNI has been to test whether serial magnetic resonance imaging (MRI), positron emission tomography (PET), other biological markers, and clinical and neuropsychological assessment can be combined to measure the progression of mild cognitive impairment $(\mathrm{MCl})$ and early Alzheimer's disease (AD). For up-to-date information, see www.adni-info.org.

The ADNI cohort information was downloaded from the ADNI data repository

137 (http://adni.loni.ucla.edu/), supplying all the demographic information, neuropsychological and clinical assessment data, and diagnostic information that was previously published (16). Prior Institutional Review Board approval was obtained at each participating institution and written informed consent was obtained for all participants. Information about the ADNI project is provided on http://www.adni-info.org/

142 and the associated publication (17).

\section{2.2. Pathology, clinical and lipidomics data}


144 Untargeted lipidomics, AD diagnosis, CSF biomarkers including Total Tau (t-tau) and

145 amyloid beta $\left(A \beta_{1-42}\right)$, cognitive decline (ADAScog13), brain atrophy represented by

146 Spatial Pattern of Abnormality for Recognition of Early Alzheimer's disease (SPARE-AD)(17)

147 data were obtained from the ADNI repository (http://www.adni-info.org/). Generation and

148 quality control of lipidomics data have been described in (18) .

149 2.3. Detection of sets of co-regulated lipids

150 A pair-wise Spearman-rank correlation matrix was generated for lipids using the $R$

151 function cor.test. The matrix was converted to a hierarchical tree model using the hclust

152 function in $\mathrm{R}$ with the ward linkage method. The resulting tree model was divided into

153 clusters using the tree cutting algorithm dynamicTreeCut (19). We used a minimum

154 cluster size of three and a split depth of four in the tree cutting method.

155 2.4. Association modeling

156 Linear regression models were established for association of serum lipid abundances

157 and CSF biomarkers and indices for cognitive decline and brain atrophy. No confounding

158 variables were included in the regression models. Logistic regression models were

159 calculated to associate serum lipids with AD diagnosis. Lipid abundances were scaled

160 by the mean substracting approach. All models were unadjusted to identify all the lipid

161 co-regulatory sets that were associated specifically with only $A D$ or with $A D$ and other

162 demographics or confounding factors. Data from all ADNI-1 participants were included

163 in the analysis.

164 2.5. Lipid set enrichment analysis

165 Co-regulatory lipid sets detected by the dynamicTreeCut method (19) were used as an

166 input for cluster enrichment analysis using the Kolmogorov-Smirnov test as described

167 in the ChemRICH method (20). In this test, the distribution of p-values was assumed to

168 be uniform under a null hypothesis for a lipid cluster. Raw p-values obtained from the 
169 linear and logistic models were used as input for computing the enrichment statistics by

170 comparing the experimental $p$-values with the uniform distribution. Set level p-values

171 were adjusted for false discovery rate using the Benjamini-Hochberg method.

\subsection{Source code}

173 All statistical analyses were performed in R programming language version 3.4.0.

174 The R-script is available at http://github.com/barupal/ADNI

175

\section{Results}

177 The main objective of the study was to identify lipid co-regulatory modules that were

178 associated with AD diagnosis and its clinical and pathological features. In this direction,

179 we first computed univariate association models and obtained raw p-values for each

180 lipid. Next, we identified lipid co-regulatory modules, which were then used as set-

definitions for a lipid-set enrichment analysis using the raw p-values for lipids. To minimize the false-negative rate on the set-level statistics, univariate $p$-values were not corrected for the multiple hypothesis testing.

\subsection{Subject cohort and lipidomics details}

185 Supplement Table 1 summarizes the details for the 806 ADNI participants at baseline included in the present study. The baseline ADNI1 serum lipidomics dataset contained 16 different lipid chemical classes summarizing 349 annotated lipids (Table 1).

\subsection{Regression models for individual lipids}

189 We first tested all individual lipids for their association with both early and late AD pathogenic markers and cognitive changes (Supplement Table S2). Raw p-values from these association models will be used as an input for the lipid set enrichment analysis in the following section. Figure 1 shows the number of significantly associated lipids in 
regression model (raw $p$-value $<0.05$ ), making it difficult to biologically interpret them on individual lipid level. Two AD phenotypes showed strong positive associations with individual serum lipids, CSF Total tau and SPARE AD. Conversely, three phenotypes were mostly negatively associated with individual blood lipids, including the two related phentoypes AD diagnosis and its major contributor, ADASCog13. Overall, AD diagnosis was associated with a decline in many lipid levels which could point to lower metabolic activity in specific lipid metabolic pathways. When analyzing all individual lipids that were associated with at least one AD-phenotype, we found a very high specificity of lipid associations with a particular AD-phenotype (Figure 2 and Table S3). 48\% (168/349) of all lipids were associated with at least one AD-phenotype (Table S3). Specifically, for known lipids, $63 \%$ of all AD-phenotype associated lipids were specific to only one phenotype and not shared with others (Figure 3). $28 \%$ of the detected associations of known lipids were shared between two phenotypes, driven by lipids shared between the two related phentoypes AD diagnosis and its major contributor, ADASCog13, in addition to lipids shared between total tau and SPARE-AD. Conversely, Abeta142 showed few shared lipids. Importantly, there was no identified lipid that was shared between four phenotypes, and only one lipid that was associated with all AD-phenotypes (arachidonyl-

211 Iysophosphatidylethanolamine; LPE C20:4). Many lipids are co-regulated by the activity 212 of specific lipases or other enzymes involved in lipid remodeling. Identifying 213 commonalities of biochemical mechanisms may lead to underlying genetic drivers or 214 environmental factors implicated in AD-etiology. Therefore, we next focused on 215 identifying sets of co-regulated lipids associated with AD pathophysiology rather than 216 interpreting individual lipids.

\section{3.3. Identifying sets of co-regulated lipids}


218 Lipid classification often uses chemical structure as the only criterion. To specify the

219 biochemical relationships between circulating blood lipids, we instead used correlation analysis to determine sets of lipids. A pair-wise Spearman correlation matrix followed by hierarchical clustering with the DynamicTreeCut dendrogram cutting method (19) yielded a total of 28 co-regulated lipid sets in the ADNI1 dataset (Figure 3). The mean size was 12.5 lipids per set, ranging from 4 to 28 members. These lipid sets (LM) were named LM1 to LM28. The average Spearman correlation coefficient rho across sets was 0.63 with a range of $0.19<r h o<0.82$. Figure 3 and Supplemental Table S2 show that some lipid co-regulatory sets were composed of lipids from the same chemical classes (such as Set-17 for free fatty acids, Set- 1 for triacylglycerides and Set-14 for ceramides) whereas other sets were heterogeneous (such as Set-3 consisting of ceramides and sphingomyelins, or Set-7 that includes phosphatidylinositols and phosphatidylcholines). Interestingly, several classes of lipids were found with distinct co-regulation within each class. For example, triacylglycerides were not found as one large group of co-regulated species, but clustered in three specific sets, and similarly, free fatty acids were found in two different sets, Set-9 consisting only of saturated fatty acids and Set-17 comprised only of unsaturated fatty acids. Similarly, other lipid classes were distributed across different sets, too. For example, phosphatidylcholines were found in five sets and sphingomyelins were co-regulated in three sets, indicating downstream regulation of lipid

237 biochemistry by specific elongases, desaturases, lipases, acyl-transferases within each 238 lipid class (Figure 2).

\section{$239 \quad 3.4$ Associating lipid sets with AD-phenotypes}

240 These lipid groups served as input for a lipid-set enrichment analysis (LSEA)(20) along 241 with the $p$-value and beta coefficient results from the regression models. Overall, 19 out 242 of 28 lipid sets were significantly associated with at least one AD-phenotype (Figure 4, 
243 Table S3) using the Kolmogorov-Smirnoff statistical test with FDR-corrections. Eight sets

244 were uniquely associated with only one specific AD-phenotype, but only one set was

245 associated with four phenotypes, Set-11, consisting primarily of ceramides and

246 phosphatidylcholines. Set-11 did not include polyunsaturated acyl chains with three or

247 more double bonds. Six sets were associated with two AD-phenotypes and four sets

248 were correlated with three AD-phenotypes, but no set correlated with all five phenotypes.

249 More than two-thirds of all associations were positively correlated between lipid sets and

250 phenotypes, mostly driven by the t-tau phenotype that also had the highest number of

251 correlated lipid sets. Conversely, ADASCog13 showed the highest number of negative

252 associations with lipid sets. We therefore investigated the individual phenotypes with

253 respect to the composition of their associated lipid sets.

\subsubsection{Lipid sets associated with AD diagnosis}

255 AD-diagnosis was significantly associated with seven distinct lipid sets (Figure 4) after

256 FDR correction. Specifically, the phenotype was highly significantly negatively correlated

257 with lipid Set-26 and Set-4. Both sets were comprised of acyl chains with at least one polyunsaturated fatty acyl chain (PUFA) (see Table S2), either eicosapentaenoic acid (EPA), docosahexaenoic acid (DHA) or arachidonic acid (AA). Set-26 consisted exclusively of triacylglycerides that also contained either EPA or DHA, but not a single saturated fatty acid. Set-4 was mixed between different phospholipid head groups, cholesteryl esters and free fatty acids, indicating that the co-regulation mechanisms focused on the modulation and incorporation of acyl chains irrespective of the lipid class.

264 Set-23 was also negatively correlated with AD-diagnosis and comprised of DHAcontaining choline- and ethanolamine-plasmalogens. Conversely, two other sets of lipids were positively associated with AD-diagnosis, most significantly for Set-5 and Set-20, 
triacylglycerols with acyl groups that did not contain any PUFA with four or more double bonds, and only one single lipid with linolenic acid (C18:3). Set-20 was exclusively composed of unsaturated choline-plasmalogens, but not containing any EPA or DHA acyl chain.

\subsubsection{Lipid sets associated with CSF $A \beta_{1-42}$}

274 CSF $A \beta_{1-42}$ was significantly associated with four lipid sets (Figure 4). Three sets were 275 negatively correlated, Set-11, Set-7 and Set-8. Set-7 was the only lipid set that contained phosphatidylinositols, in addition to phosphatidyl cholines. Acyl chains were primarily saturated or mono- and di-unsaturated. Similarly, Set-8, consisted mostly of desaturated acyl groups with less than four double bonds, exclusively found as lysophosphatidylcholines. In the same way, no PUFA-acyl chains were found in Set-11, a heterogenous set of ceramides and choline-plasmalogens. Importantly, the only positive association of a lipid set with CSF $A \beta_{1-42}$ was Set-26 that was completely made of PUFAtriacylglycerides.

\subsubsection{Lipid sets associated with CSF tau}

CSF total tau correlated with 12 lipid sets, the highest number of associated lipid sets among all phenotypes (Figure 4). All sets except Set-1 were positively correlated with

287 CSF-total tau. Three unique sets that were not shared with other phenotypes. Set-16 was composed of acylcarnitines with increasing degree of desaturation, and Set-17 was a set of monounsaturated fatty acids. Set-1 was less significant in comparison to other set associations. Four sets were shared with brain atrophy, four sets were shared with AD-diagnosis, two sets with amyloid beta and four lipid sets were shared with cognitive decline. Notably, set-19 was also associated with brain atrophy and contained mostly 
293 EPA-side chain phosphatidylcholines. Set-3 was composed of sphingomyelins and ceramides that were not associated with diagnosis or amyloid beta, but instead was also linked with cognitive decline and SPARE-AD.

296

\subsubsection{Lipid sets associated with Brain atrophy (SPARE-AD)}

298 Brain atrophy was most significantly associated with Set 27, 19, 11 and 2 (Figure 4). 299 Three sets (Set 2, Set 11, Set 27) were void of PUFA-side chains with either 300 phosphoplipid or sphingolipid head groups. Conversely, Set 19 contained mostly EPA301 side chain phosphatidylcholines and was further associated with CSF total tau. Similarly,

302 Set 21 was associated with both phenotypes, containing phospholipids and their lyso303 forms with the PUFA acyl chain arachidonic acid.

304

305

\subsubsection{Lipid sets associated with cognitive functions}

Most of the lipid sets associated with cognitive decline were also associated with AD

307 diagnosis (Figure 4). Additionally, it was negatively associated with set Set-22 which consisted of ethanolamine-plasmalogens.

\section{Discussion}

311 We here focused on associations between blood lipids and five AD-phenotypes guided 312 by known contributions of lipids and metabolic co-morbidities to Alzheimer's disease. We 313 systematically tested both the association of individual lipids and the association with 314 sets of co-regulated lipids. This approach showed an important advantages over 315 previous "feature" based lipidomics-AD studies (9), (21) that did not focus on specific 316 lipid groups, their side chains or their biological regulation. Without clear lipid 317 identification, feature-based associations miss biological insights and have a high risk of 
not being validated in subsequent studies because each individual lipid may cause more

319 than one feature in LC-MS based lipidomics $(21,22)$. Instead we used the largest major lipid classes, identified by extensive MS/MS fragmentation analysis (23) and enabling analyses reaching to the level of acyl chains. A second difference to previous efforts was a focus on summarizing lipids by statistical co-regulation instead of only relying on univariate analysis or grouping by lipid head groups. This expansion of classic statistical analysis was critical to extend from diagnostic biomarkers (that need correctiong for multiple testing using false discovery rate (FDR) adjustments) to revealing underlying biological mechanisms. The axiom of univariate analyses, the mutual independence of variables, is untrue in lipid biology. Moreover, stringent FDR corrections lead to an increased number of false negative results and compromise the statistical power to investigate the biological mechanisms and pathways. As lipids are poorly presented in biochemical pathway databases (20), classic metabolic pathway 332 enrichment analysis (24) ignores a majority of detected lipids and is unsuitable for 333 lipidomics. Instead, Spearman-rank correlation based matrices yielded specific clusters 334 of lipids associated with Alzheimer's disease phenotypes by using the robust 335 Kolmogorov-Smirnov test for $p$-value distributions. These lipid sets showed very distinct metabolic features that we identified as preferential use of specific polyunsaturated fatty

337 acids that replaced saturated or monounsaturated fatty acids for distinct lipid classes.

338 These mechanisms of lipid desaturation, elongation, and acyl-chain remodeling were 339 disturbed in early and later stages of Alzheimer disease. A minimal overlap among lipid 340 sets was observed (Figure 3) with respect to statistical associations with AD phenotypes, 341 indicating that quite distinct lipid biochemical processes were involved in the early and 342 later stages of AD. Lipid metabolic pathways associated with the early stage, in 
343 particular, may provide new therapeutic targets to stop AD progression. MUFA-

344 containing lipids were positively associated with the brain atrophy and tau accumulation

345 whereas PUFA-containing lipids were negatively associated with AD diagnosis and 346 cognitive decline. Therapeutic strategies targeting MUFA lipid pathways at the early 347 stages of AD could therefore be potentially more effective in delaying the progression of 348 the disease.

\section{$349 \quad 4.1$ Lipids linked to the amyloid beta clearance pathway}

350 A decrease in the $\mathbf{C S F} \mathbf{A} \boldsymbol{\beta}_{1-42}$ peptide marker is indicating a poor clearance of the peptide 351 in the brain, leading to its extra-neuronal accumulation. In our study, poor clearance was 352 indicated by negative associations with lipids sets, including sets that contained 353 phosphatidylinositols, lysoPCs, ceramides and choline-plasmalogens and PUFA TGs.

354 The amyloid $\beta$ peptide is known to cause mitochondrial dysfunction (25) which can lead 355 to neurodegeneration via autophagic cascades $(26,27)$. The associated lipids, specifically ceramides and phosphatidylinositols and lysoPCs have been linked with cell death and may also contribute in the Amyloid Beta mediated toxicity in neurons (28-30). Higher levels of ceramides containing oleic acid (C18:1) have been shown to increase $A D$ risk $(4,5)$. We validate this finding in our study and also observed lower levels of phosphoinositols containing polyunsaturated fatty acids to correlate with poor Amyloidbeta clearance. An alternative explanation to our data is an impaired amyloid beta 362 clearance in the liver(31)that subsequently leads to dysregulation of lipid metabolism in 363 the liver. Overall, our data suggest that these lipid sets can serve as serum biomarkers for disturbed Amyloid beta pathway regulation in brain and can complement Amyloid beta imaging assays.

\subsection{Cerebrospinal fluid total tau}


368 CSF tau is a marker for accumulating tau tangles in the brain tissues, causing neurodegeneration. We found that the total CSF tau marker was significantly associated with lipid sets enriched in monounsaturated fatty acids, acyl-carnitines, ceramides, sphingomyelins, and EPA containing phosphatidylcholines. Increased fatty acids and

372 acyl-carnitines are known markers of impaired fatty acid beta oxidation in 373 mitochondria(32), specially during metabolic diseases such as diabetes and obesity(33, $37434)$. We found free fatty acids and acylcarnitines to be positively correlated with total tau, 375 supporting the notion of tau mediated neurodegeneration and mitochondrial 376 dysfunctions. Mitochondrial impairment was further evidenced by positive associations 377 of total tau with sets of ceramides, because accumulating ceramide are known to induce 378 cell death and to increase the AD risk in normal subjects (5) Rozen et al. 2011. Higher 379 ceramide levels were also reported for early stage Alzheimer's disease (35-37). These findings indicate that these lipids may be involved in early neurodegenerative pathways, and their underlying pathways might lead to candidates for new therapeutic strategies.

\subsection{Lipid sets linked with brain atrophy}

SPARE-AD is a composite index of brain atrophy and indicates the neurodegeneration magnitude. We found a high overlap of lipid sets that were associated with both SPARE-AD and total tau, reinforcing the usability of these serum lipids as biomarkers for neurodegeneration. These lipid sets included phosphatidylcholines and sphingolipids that were enriched in polyunsaturated fatty acyls (PUFA) eicosapentaenoic acid and arachidonic acid (EPA, AA). These fatty acids are main components of brain lipids $(38,39)$. The loss of brain tissue may cause an increase in levels of serum lipids that include EPA and AA as acyl groups through lipid remodelling $(40,41)$. We here identify lipid pathways associated with tau-mediated brain atrophy

\section{2 that eventually contributes to AD.}




\subsection{AD diagnosis and cognitive decline}

395 Previous publications reported that lower levels of PUFA in AD subjects across multiple 396 lipid classes, along with higher levels of monounsaturated lipids (4, 8, 9, 42-46). We 397 found numerous, very significant associations of omega-3 and omega- 6 containing 398 complex lipids with AD diagnosis and cognitive functions. Our analysis is consistent with 399 these results as shown by AD associated lipids in Set-4, Set-20, Set-23 (Figure 4). We 400 here specify that the major difference is not related to total levels of mono- or 401 polyunsaturated fatty acids, but the extent at which these fatty acids are incorporated 402 into different complex lipids. Clear differences in circulating PUFA phospholipid and 403 PUFA triacylglycerol levels in $A D$ subjects in comparison to normal subjects were 404 observed, likely due to dysregulation of biosynthesis in liver. Here, substrate preference 405 of MGAT and DGAT enzymes in the liver may play an important role, but the exact 406 specificities of acyltransferase enzymes (and their corresponding lipase enzymes) are not well studied. Levels of anti-inflammatory plasmalogens (47), important lipids for brain 408 membrane functions $(45,48)$, were decreased in $A D$ patients in comparison to 409 cognitively normal subjects. Lower levels of plasmalogens have been linked to AD (45). 410 However, in clinical trials, EPA and DHA supplementation do not improve the cognitive 411 function of AD subjects (49). Nutritional intervention trials such as the European 412 LipiDiDiet have failed to show any cognitive improvement in AD subjects. A broad413 spectrum effect of FOS on additional lipid pathways may explain the failure of this trial 414 and warrants further lipidomics studies for serum specimens of this trial's participants. It 415 was observed that the incorporation of omega- 6 fatty acids was increased in $A D$ 416 subjects. These fatty acids are well-known precursors to pro-inflammatory molecules 417 such as leukotrienes. Further studies are needed to test if post-mortem brain tissues of 
418 AD subjects show similar disruption in fatty acid incorporation and if these patterns 419 correlate with AD severity or other AD phenotypes.

420

421

\section{Conclusions}

422 Using co-regulated sets of lipids enabled us to find significant associations of lipids with 423 Alzheimer's disease that led to biochemical mechanisms. Across the spectrum of AD progression, pathways were dysregulated that pointed to lipid desaturation, elongation and remodelling. These pathways provide new targets as well candidate biomarkers for the population screening for AD prevention. Future studies are needed to tease out the roles of genetic variations, drug, and diet the metabolism of MUFA and PUFAs and their complex lipids and their roles in AD.

\section{Acknowledgments}

\section{$431 \quad$ Funding}

432 This work was funded through NIH awards U54 Al138370, U19 AG023122 and U2C ES030158.

433 National Institute on Aging (R01AG046171, RF1AG051550, and RF1AG057452 and 434 3U01AG024904-09S4) supported the Alzheimer Disease Metabolomics Consortium which is a 435 part of NIA national initiatives AMP-AD and M2OVE AD. Data collection and sharing for this 436 project was funded by the Alzheimer's Disease Neuroimaging Initiative (ADNI) (National 437 Institutes of Health Grant U01 AG024904) and DOD ADNI (Department of Defense award number W81XWH-12-2-0012). ADNI is funded by the National Institute on Aging, the National 439 Institute of Biomedical Imaging and Bioengineering, and through generous contributions from 440 the following: AbbVie, Alzheimer's Association; Alzheimer's Drug Discovery Foundation; Araclon 441 Biotech; BioClinica, Inc.; Biogen; Bristol-Myers Squibb Company; CereSpir, Inc.; Cogstate; Eisai 442 Inc.; Elan Pharmaceuticals, Inc.; Eli Lilly and Company; Eurolmmun; F. Hoffmann-La Roche Ltd 443 and its affiliated company Genentech, Inc.; Fujirebio; GE Healthcare; IXICO Ltd.; Janssen 
444 Alzheimer Immunotherapy Research \& Development, LLC.; Johnson \& Johnson Pharmaceutical

445 Research \& Development LLC.; Lumosity; Lundbeck; Merck \& Co., Inc.; Meso Scale Diagnostics,

446 LLC.; NeuroRx Research; Neurotrack Technologies; Novartis Pharmaceuticals Corporation;

447 Pfizer Inc.; Piramal Imaging; Servier; Takeda Pharmaceutical Company; and Transition

448 Therapeutics. The Canadian Institutes of Health Research is providing funds to support ADNI

449 clinical sites in Canada. Private sector contributions are facilitated by the Foundation for the

450 National Institutes of Health (www.fnih.org). The grantee organization is the Northern California

451 Institute for Research and Education, and the study is coordinated by the Alzheimer's

452 Therapeutic Research Institute at the University of Southern California. ADNI data are

453 disseminated by the Laboratory for Neuro Imaging at the University of Southern California.

\section{Author's contribution}

455 DKB and OF generated the lipidomics dataset. DKB, RB, RKD and OF design the study. 456 DKB and SF performed the statistical analyses. AJS, PJM, MA and KN contributed in 457 data interpretation. All author contributed in the manuscript writing.

458 Transparency declaration: Authors affirms that the manuscript is an honest, accurate, 459 and transparent account of the study being reported; no important aspects of the study 460 have been omitted; and any discrepancies from the study as planned have been 461 explained. 
Figure legends

464 Figure 1. Co-regulated sets of serum lipids in the ADNI lipidomics dataset. Sets

465 were detected by the dynamicTreeCut algorithm (see method). Node colors show

466 different chemical classes. FA - fatty acids, AC - acyl carnitines, PC

467 phosphatidylcholines, CE - cholesteryl esters, SM - sphingomyelins, Cer - ceramides,

468 PE - phosphatidylethanolamines, TG - triacylglycerols, PI - phosphatidylinositols, DG

469 - diacylglycerols, LPC - lysophosphatidylcholines.

Figure 2. Number of significantly different lipids with AD phenotypes in

univariate statistics. Directions of beta coefficients in regression models are given by

colors as blue (negative) and red (positive) associations using uncorrected $p<0.05$

473 values. $\mathrm{CN}$ : cognitively normal, $\mathrm{LMCl}$ : late mild cognitive impairment, $\mathrm{AD}$ : Alzheimer's

474 disease. DIAG : linear models for diagnosis, tau - linear model for tau, $A \beta_{1-42}$ - linear model for amyloid beta peptide 42, SPARE-AD - linear model for Spatial Pattern of

Abnormality for Recognition of Early Alzheimer's disease index, ADASCog13 -

477 Cognitive Subscale of the Alzheimer's Disease Assessment Scale index.

479 Figure 3. Number of significantly associated lipids across AD-phenotypes.

480 Uncorrected $p<0.05$ values for five AD-phenotypes. DIAG : linear models for 481 diagnosis, tau - linear model for tau, ABETA142 - linear model for amyloid beta 482 peptide 42, SPARE-AD - linear model for Spatial Pattern of Abnormality for 483 Recognition of Early Alzheimer's disease index, ADASCog13 - Cognitive Subscale of the Alzheimer's Disease Assessment Scale index. 
485 Figure 4. Co-regulated sets of lipids significantly associated with AD-

486 phenotypes. Direction of associations is given by red (positive) and blue (negative)

487 edge colors. Line thickness indicates the significance of associations (see

488 Supplementary Table 4 for details). Lipid compositions for each set are shown in

489 Figure 1 and Supplementary Table S1. DIAG : linear models for diagnosis, tau - linear

490 model for tau, ABETA142 - linear model for amyloid beta peptide 42, SPARE-AD -

491 linear model for Spatial Pattern of Abnormality for Recognition of Early Alzheimer's

492 disease index, ADASCog13 - Cognitive Subscale of the Alzheimer's Disease

493 Assessment Scale index. 


\section{Tables}

496 Table 1. Lipid classes and sub-classes in the ADNI serum lipidomics untargeted

497 dataset

\begin{tabular}{|c|c|c|}
\hline Class & Subclass & Count \\
\hline Acylcarnitine (AC) & Acylcarnitine & 9 \\
\hline Free fatty acid (FA) & Fatty acid & 29 \\
\hline \multirow[t]{2}{*}{ Sterol lipids } & Cholesterol & 1 \\
\hline & Cholesteroyl ester (CE) & 8 \\
\hline \multirow[t]{7}{*}{ Phospholipid } & Lysophosphatidylcholine (LPC) & 22 \\
\hline & Lysophosphatidylethanolamine (LPE) & 4 \\
\hline & Phosphatidylcholine (PC) & 53 \\
\hline & Phosphatidylethanolamine (PE) & 11 \\
\hline & Phosphatidylinositol (PI) & 11 \\
\hline & Plasmalogen phosphatidylcholine (p-PC) & 28 \\
\hline & Plasmalogen phosphatidylethanolamine (p-PE) & 15 \\
\hline \multirow[t]{3}{*}{ Sphingolipid } & Ceramide (CER) & 19 \\
\hline & Glucosylceramide (GluCer) & 8 \\
\hline & Sphingomyelin (SM) & 34 \\
\hline \multirow[t]{2}{*}{ Acylglycerols } & Diacylglycerol (DG) & 13 \\
\hline & Triacylglycerol (TG) & 84 \\
\hline
\end{tabular}




\section{References}

502

503

504

505

506

507

508

509

510

511

512

513

514

515

516

517

518

519

520

521

522

523

524

525

526

527

528

529

530

531

532

533

534

535

536

537

538

539

540

541

542

543

544

545

546

547

548

1. $\quad$ Scheltens P, et al. (2016) Alzheimer's disease. Lancet 388(10043):505-517.

2. Liu CC, Liu CC, Kanekiyo T, Xu H, \& Bu G (2013) Apolipoprotein E and Alzheimer disease: risk, mechanisms and therapy. Nature reviews. Neurology 9(2):106-118.

3. Bressler J, et al. (2017) Metabolomics and cognition in African American adults in midlife: the atherosclerosis risk in communities study. Translational psychiatry 7(7):e1173.

4. Han X, et al. (2011) Metabolomics in early Alzheimer's disease: identification of altered plasma sphingolipidome using shotgun lipidomics. PloS one 6(7):e21643.

5. Mielke MM, et al. (2012) Serum ceramides increase the risk of Alzheimer disease: the Women's Health and Aging Study II. Neurology 79(7):633-641.

6. Schilling S, et al. (2017) Differential associations of plasma lipids with incident dementia and dementia subtypes in the $3 C$ Study: A longitudinal, population-based prospective cohort study. PLoS medicine 14(3):e1002265.

7. van der Lee SJ, et al. (2018) Circulating metabolites and general cognitive ability and dementia: Evidence from 11 cohort studies. Alzheimer's \& dementia : the journal of the Alzheimer's Association 14(6):707-722.

8. Proitsi P, et al. (2017) Association of blood lipids with Alzheimer's disease: A comprehensive lipidomics analysis. Alzheimer's \& dementia : the journal of the Alzheimer's Association 13(2):140-151.

9. Proitsi P, et al. (2015) Plasma lipidomics analysis finds long chain cholesteryl esters to be associated with Alzheimer's disease. Translational psychiatry 5:e494.

10. Varma VR, et al. (2018) Brain and blood metabolite signatures of pathology and progression in Alzheimer disease: A targeted metabolomics study. PLoS medicine 15(1):e1002482.

11. Toledo JB, et al. (2017) Metabolic network failures in Alzheimer's disease: A biochemical road map. Alzheimer's \& dementia : the journal of the Alzheimer's Association 13(9):965-984.

12. Mapstone $M$, et al. (2014) Plasma phospholipids identify antecedent memory impairment in older adults. Nature medicine 20(4):415-418.

13. Fiandaca MS, et al. (2015) Plasma 24-metabolite Panel Predicts Preclinical Transition to Clinical Stages of Alzheimer's Disease. Front Neurol 6:237.

14. Casanova R, et al. (2016) Blood metabolite markers of preclinical Alzheimer's disease in two longitudinally followed cohorts of older individuals. Alzheimer's \& dementia : the journal of the Alzheimer's Association 12(7):815-822.

15. Xu M, et al. (2018) A systematic integrated analysis of brain expression profiles reveals YAP1 and other prioritized hub genes as important upstream regulators in Alzheimer's disease. Alzheimer's \& dementia : the journal of the Alzheimer's Association 14(2):215-229.

16. Toledo JB, et al. (2017) Metabolic network failures in Alzheimer's disease-A biochemical road map. Alzheimer's \& dementia : the journal of the Alzheimer's Association.

17. Toledo JB, et al. (2013) Relationship between plasma analytes and SPARE-AD defined brain atrophy patterns in ADNI. PloS one 8(2):e55531.

18. Barupal DK, et al. (2018) Generation and quality control of lipidomics data for the alzheimer's disease neuroimaging initiative cohort. Sci Data 5:180263.

19. Langfelder $P$, Zhang B, \& Horvath $S$ (2008) Defining clusters from a hierarchical cluster tree: the Dynamic Tree Cut package for R. Bioinformatics 24(5):719-720.

20. Barupal DK \& Fiehn O (2017) Chemical Similarity Enrichment Analysis (ChemRICH) as alternative to biochemical pathway mapping for metabolomic datasets. Sci Rep 7(1):14567.

21. Mahieu NG \& Patti GJ (2017) Systems-Level Annotation of a Metabolomics Data Set Reduces 25000 Features to Fewer than 1000 Unique Metabolites. Analytical chemistry 89(19):1039710406. 
22. Bowden JA, et al. (2017) Harmonizing lipidomics: NIST interlaboratory comparison exercise for lipidomics using SRM 1950-Metabolites in Frozen Human Plasma. Journal of lipid research 58(12):2275-2288.

552

553

554

555

556

557

558

559

560

561

562

563

564

565

566

567

568

569

570

571

572

573

574

575

576

577

578

579

580

581

582

583

584

585

586

587

588

589

590

591

592

593

594

595

596

597

23. Kind T, et al. (2013) LipidBlast in silico tandem mass spectrometry database for lipid identification. Nat Methods 10(8):755-758.

24. Xia J \& Wishart DS (2010) MetPA: a web-based metabolomics tool for pathway analysis and visualization. Bioinformatics 26(18):2342-2344.

25. Mossmann D, et al. (2014) Amyloid-beta peptide induces mitochondrial dysfunction by inhibition of preprotein maturation. Cell metabolism 20(4):662-669.

26. Lin MT \& Beal MF (2006) Mitochondrial dysfunction and oxidative stress in neurodegenerative diseases. Nature 443(7113):787-795.

27. Suomalainen A \& Battersby BJ (2017) Mitochondrial diseases: the contribution of organelle stress responses to pathology. Nature reviews. Molecular cell biology.

28. Elliott MR \& Ravichandran KS (2016) The Dynamics of Apoptotic Cell Clearance. Dev Cell 38(2):147-160.

29. Magtanong L, Ko PJ, \& Dixon SJ (2016) Emerging roles for lipids in non-apoptotic cell death. Cell Death Differ 23(7):1099-1109.

30. Wong MW, et al. (2017) Dysregulation of lipids in Alzheimer's disease and their role as potential biomarkers. Alzheimer's \& dementia : the journal of the Alzheimer's Association.

31. Maarouf CL, et al. (2018) Impaired hepatic amyloid-beta degradation in Alzheimer's disease. PloS one 13(9):e0203659.

32. Ciavardelli D, et al. (2016) Medium-chain plasma acylcarnitines, ketone levels, cognition, and gray matter volumes in healthy elderly, mildly cognitively impaired, or Alzheimer's disease subjects. Neurobiology of aging 43:1-12.

33. Adams SH, et al. (2009) Plasma acylcarnitine profiles suggest incomplete long-chain fatty acid beta-oxidation and altered tricarboxylic acid cycle activity in type 2 diabetic African-American women. J Nutr 139(6):1073-1081.

34. Serra D, Mera P, Malandrino MI, Mir JF, \& Herrero L (2013) Mitochondrial fatty acid oxidation in obesity. Antioxid Redox Signal 19(3):269-284.

35. Han X, D MH, McKeel DW, Jr., Kelley J, \& Morris JC (2002) Substantial sulfatide deficiency and ceramide elevation in very early Alzheimer's disease: potential role in disease pathogenesis. Journal of neurochemistry 82(4):809-818.

36. He X, Huang Y, Li B, Gong CX, \& Schuchman EH (2010) Deregulation of sphingolipid metabolism in Alzheimer's disease. Neurobiology of aging 31(3):398-408.

37. Cutler RG, et al. (2004) Involvement of oxidative stress-induced abnormalities in ceramide and cholesterol metabolism in brain aging and Alzheimer's disease. Proceedings of the National Academy of Sciences of the United States of America 101(7):2070-2075.

38. Dyall SC (2015) Long-chain omega-3 fatty acids and the brain: a review of the independent and shared effects of EPA, DPA and DHA. Front Aging Neurosci 7:52.

39. Martin V, et al. (2010) Lipid alterations in lipid rafts from Alzheimer's disease human brain cortex. Journal of Alzheimer's disease : JAD 19(2):489-502.

40. Hashidate-Yoshida T, et al. (2015) Fatty acid remodeling by LPCAT3 enriches arachidonate in phospholipid membranes and regulates triglyceride transport. Elife 4.

41. Wang B, et al. (2016) Intestinal Phospholipid Remodeling Is Required for Dietary-Lipid Uptake and Survival on a High-Fat Diet. Cell metabolism 23(3):492-504.

42. Iuliano L, et al. (2013) Plasma fatty acid lipidomics in amnestic mild cognitive impairment and Alzheimer's disease. Journal of Alzheimer's disease : JAD 36(3):545-553.

43. Cunnane SC, et al. (2012) Plasma and brain fatty acid profiles in mild cognitive impairment and Alzheimer's disease. Journal of Alzheimer's disease : JAD 29(3):691-697. 
44. Conquer JA, Tierney MC, Zecevic J, Bettger WJ, \& Fisher RH (2000) Fatty acid analysis of blood plasma of patients with Alzheimer's disease, other types of dementia, and cognitive impairment. Lipids 35(12):1305-1312.

601

45. Wood PL, et al. (2010) Circulating plasmalogen levels and Alzheimer Disease Assessment ScaleCognitive scores in Alzheimer patients. J Psychiatry Neurosci 35(1):59-62. predict cognitive decline and hippocampal volume loss. Alzheimer's \& dementia : the journal of the Alzheimer's Association 6(5):378-385.

47. Rasmiena AA, et al. (2015) Plasmalogen modulation attenuates atherosclerosis in ApoE- and ApoE/GPx1-deficient mice. Atherosclerosis 243(2):598-608.

609

610

611

612

613

48. Scott BL \& Bazan NG (1989) Membrane docosahexaenoate is supplied to the developing brain and retina by the liver. Proceedings of the National Academy of Sciences of the United States of America 86(8):2903-2907.

49. Mazereeuw G, Lanctot KL, Chau SA, Swardfager W, \& Herrmann N (2012) Effects of omega-3 fatty acids on cognitive performance: a meta-analysis. Neurobiology of aging 33(7):1482 e14171429. 
Figure 1

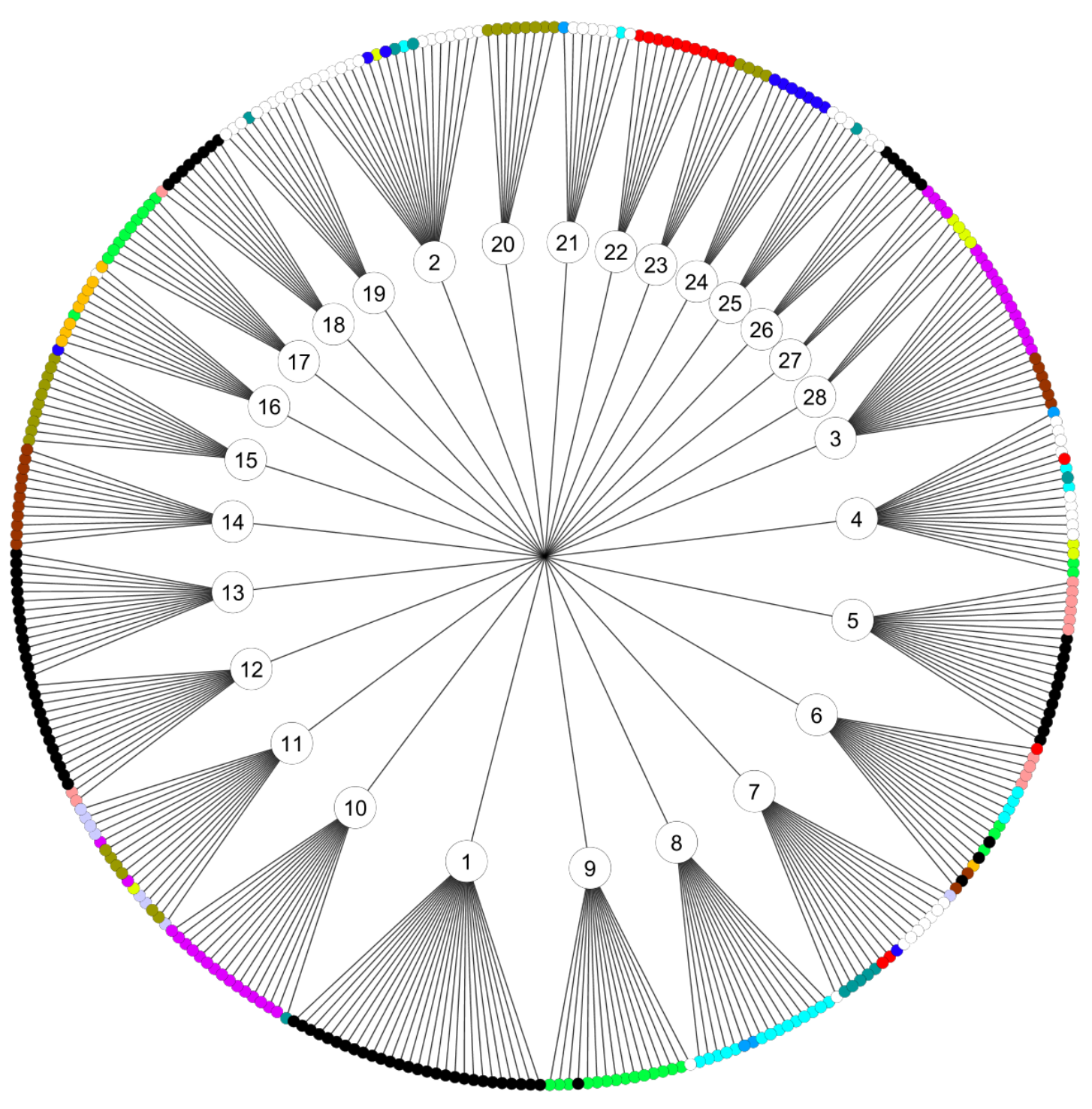

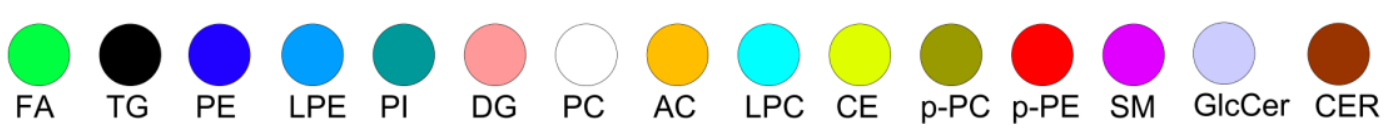


Figure 2
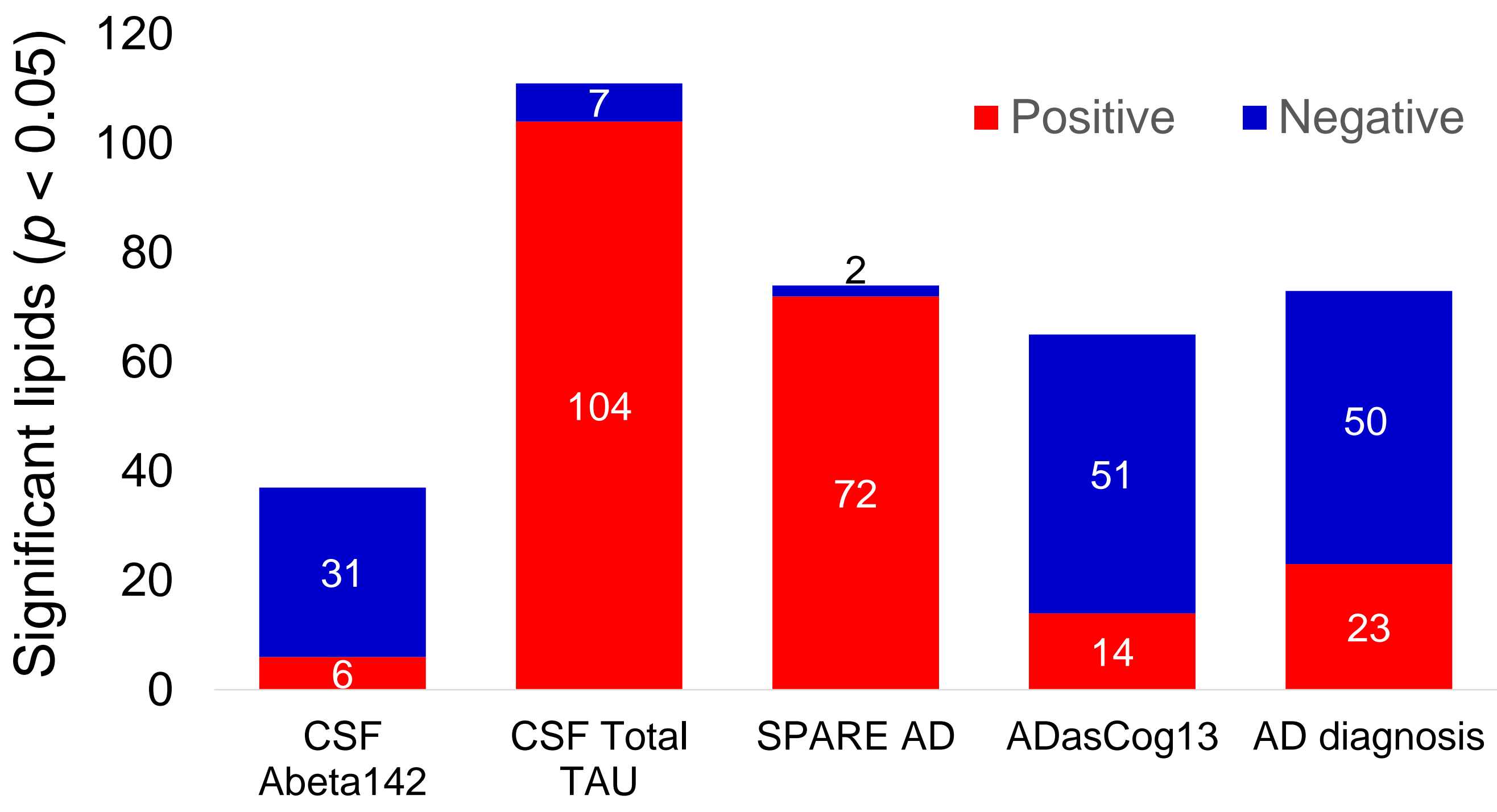
Figure 4

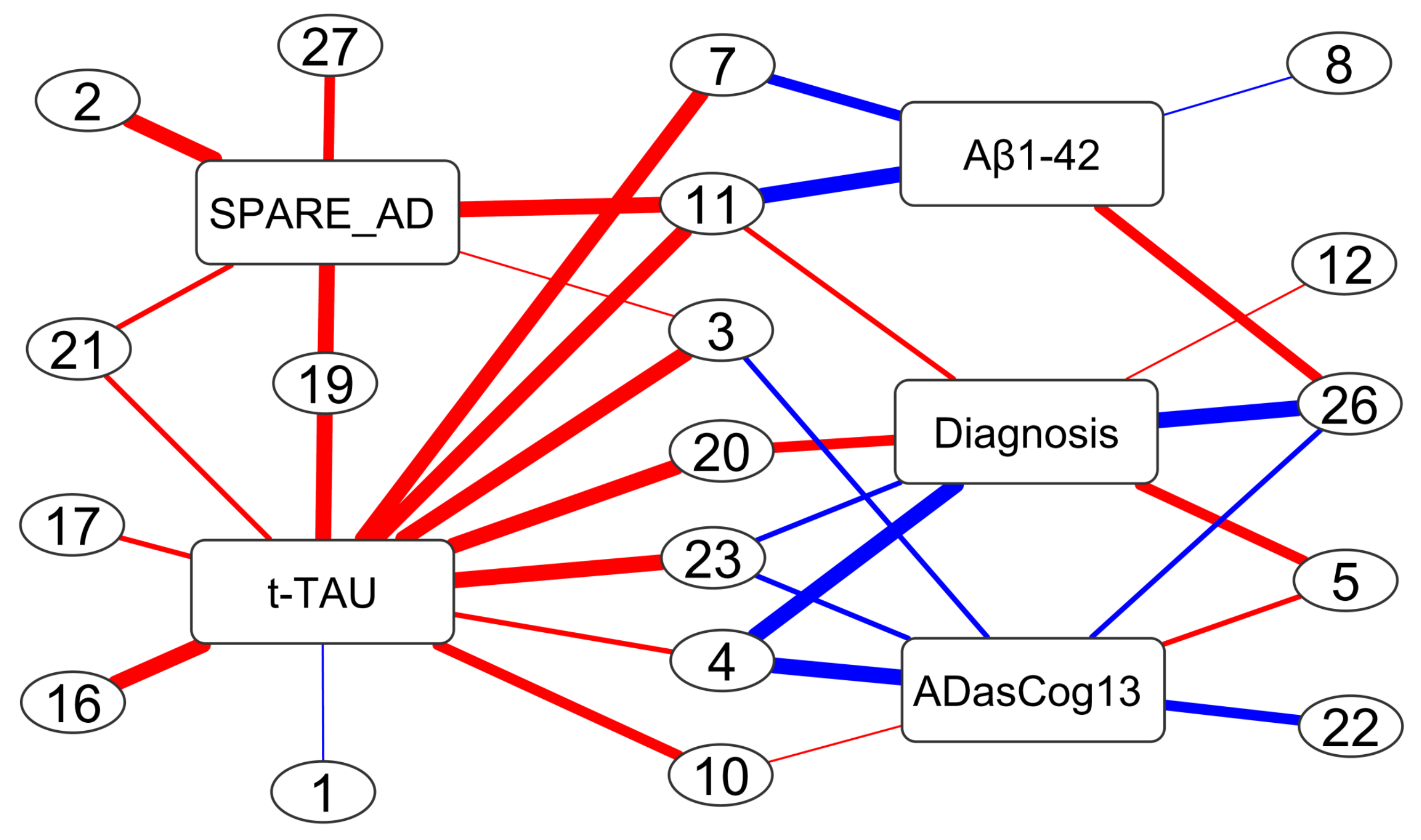

